Research Article

\title{
Novel Antibiotic Combinations of Diverse Subclasses for Effective Suppression of Extensively Drug-Resistant Methicillin-Resistant Staphylococcus aureus (MRSA)
}

\author{
Shumyila Nasir $\mathbb{D}^{1},{ }^{1}$ Muhammad Sufyan Vohra ${ }^{D},{ }^{1}$ Danish Gul ${ }^{D},{ }^{1}$ Umm E Swaiba $\mathbb{D}^{1},{ }^{1}$ \\ Maira Aleem $\bowtie{ }^{1},{ }^{1}$ Khalid Mehmood $\odot{ }^{2}$ and Saadia Andleeb $\otimes^{1}$ \\ ${ }^{1}$ Department of Industrial Biotechnology, Atta-ur-Rahman School of Applied Biosciences (ASAB), \\ National University of Sciences and Technology (NUST), Islamabad 44000, Pakistan \\ ${ }^{2}$ Department of Pharmacy, Abbottabad University of Science and Technology, Havelian, Pakistan \\ Correspondence should be addressed to Saadia Andleeb; saadiamarwat@yahoo.com
}

Received 16 June 2020; Revised 5 October 2020; Accepted 15 October 2020; Published 29 October 2020

Academic Editor: Faham Khamesipour

Copyright (c) 2020 Shumyila Nasir et al. This is an open access article distributed under the Creative Commons Attribution License, which permits unrestricted use, distribution, and reproduction in any medium, provided the original work is properly cited.

\begin{abstract}
The emergence of multidrug-resistant pathogens such as methicillin-resistant Staphylococcus aureus (MRSA), the chief etiological agent for a range of refractory infections, has rendered all $\beta$-lactams ineffective against it. The treatment process is further complicated with the development of resistance to glycopeptides, primary antibiotics for treatment of MRSA. Antibiotic combination therapy with existing antimicrobial agents may provide an immediate treatment option. Minimum inhibitory concentrations (MICs) of 18 different commercially available antibiotics were determined along with their 90 possible pairwise combinations and 64 triple combinations to filter out 5 best combinations. Time-Kill kinetics of these combinations were then analyzed to find collateral bactericidal combinations which were then tested on other randomly selected MRSA isolates. Among the top 5 combinations including levofloxacin-ceftazidime; amoxicillin/clavulanic acid-tobramycin; amoxicillin/clavulanic acidcephradine; amoxicillin/clavulanic acid-ofloxacin; and piperacillin/tazobactam-tobramycin, three combinations were found to be collaterally effective. Levofloxacin-ceftazidime acted synergistically in $80 \%$ of the tested clinical MRSA isolates. First-line $\beta$-lactams of lower generations can be used effectively against MRSA infection when used in combination. Antibiotics other than glycopeptides may still work in combination.
\end{abstract}

\section{Introduction}

Hospital-acquired methicillin-resistant Staphylococcus aureus (MRSA) has been a predominant agent for skin and nosocomial infections for several years [1-3]. MRSA is responsible for about more than $20 \%$ of all bloodstream infections, and mortality rate is as high as $25-50 \%[4,5]$. Methicillin resistance in Staphylococcus aureus is mediated by SCCmec gene, which encodes polypeptide penicillinbinding-protein 2a (PBP2a) $[6,7]$ and also provides insertion sites for plasmids and transposons which assist in transmission of resistance to non- $\beta$-lactam antibiotics $[8,9]$. Emergence of $\beta$-lactamase-producing MRSA strains has rendered all $\beta$-lactams ineffective against its infections. Glycopeptides (mainly vancomycin) remain the major class of antibiotics for treatment of MRSA, but indiscriminate use of these antimicrobial agents has led to the emergence of vancomycin-resistant $S$. aureus (VRSA) [10]. Development of resistance and other complications such as infection recurrence, and treatment failure pose a serious hindrance in treatment of MRSA infections [4, 11]. Therefore, potential options for treatment of these refractory infections need to be explored. Since other therapeutic interventions such as phage therapies are still in development, antibiotic combination therapy with existing antimicrobial agents provides an immediate treatment option. 
Usage of multidrug combinations has proven successful against infection caused by $H$. pylori, M. tuberculosis, A. baumannii, P. aeruginosa, and K. pneumonia [12-16]. Historically, vancomycin-aminoglycoside combination was successfully employed for endocarditis caused by MRSA, but due to increased possibility of renal impairment, this combination is not recommended anymore [17-19]. More recent studies, where vancomycin was used concurrently with beta-lactams improved in vitro results, were observed. In another study, antibiotics from different subclasses and generations of $\beta$-lactams (meropenem, piperacillin, and tazobactam) were successfully combined against MRSA infections [20].

In this study, we combined FDA approved antibiotics from different generations and classes to determine synergistic combinations which do not necessarily include vancomycin (or any other glycopeptide) against highly resistant MRSA. We anticipate that using antibiotic combinations of commercially available antibiotics from diverse subclasses has a potential for overcoming antibiotic-resistant infections and may serve as a powerful technique in reversing antibiotic resistance on top of producing bactericidal effects.

\section{Materials and Methods}

Isolates of $S$. aureus were acquired from major hospitals of Rawalpindi, Lahore, and Peshawar, and then screened for methicillin and vancomycin resistance. Further susceptibility testing by the Kirby disk diffusion method was performed, and by using clinical breakpoints from the Clinical and Laboratory Standards Institute (CLSI), an extensively drug-resistant (XDR) MRSA isolate (LR-2) was selected for antibiotic synergy testing [21]. A total of 18 different commercially available antibiotics (Table 1) were used for 90 possible pairwise combinations (Figure 1(a)) and 64 triple combinations (antibiotic from varying class/penicillin/inhibitor) (Figure 2(a)). Each experiment was conducted in duplicate, and mean values were calculated for each combination.

XDR MRSA isolate was cultured in cation-adjusted Muller-Hinton Broth (MHB) for 24 hours at $37^{\circ} \mathrm{C}$. Minimum inhibitory concentration (MIC) was determined by the broth microdilution method as described by Wiegand et al. [22]. Stock solutions of antimicrobial agents were prepared by calculating the amount of antibiotic to be used: (amount of drug in each tablet $(\mathrm{y})) / 10 \mathrm{mg}=($ amount of active agent in each tablet)/(amount of drug needed to produce $10 \mathrm{mg} / \mathrm{ml}$ solution $(\mathrm{x})$ ).

The antibiotic-containing media were serially diluted in 96-well plate to create an array of 10-fold dilution across the row. Log-phase culture of MRSA was diluted in MHB to produce $1 / 50$ dilution of bacterial suspension and inoculated in the 96-well plate. Column 11 and 12 were kept as negative and positive control, respectively. Plates were incubated for 24 hours at $37^{\circ} \mathrm{C}$. Wells showing no visible turbidity after $24 \mathrm{~h}$ were subcultured on plate count agar for colony counting. Minimum inhibitory concentration (MIC) was considered as the lowest concentration inhibiting 99\% of bacterial growth. Each experiment was performed in duplicate. Synergistic combinations were determined by the fractional inhibitory concentration index (FICI) method [23-26]. FICI calculation involves MIC of the antimicrobial agents in combination divided by the MIC of the agents alone to determine antibiotic interactions, as shown in the following formula:

$$
\mathrm{FICI}=\frac{\operatorname{MIC}_{(\mathrm{comb} A B)}}{\mathrm{MIC}_{(A)}}+\frac{\mathrm{MIC}_{(\operatorname{comb} 6 B)}}{\mathrm{MIC}_{(B)}},
$$

whereby FICI $\leq 0.5$ was interpreted as "synergistic," FICI $=1-4.0$ was considered "indifferent," and FICI $>4.0$ was inferred "antagonistic."

For further analyses, five highly synergistic combinations against MRSA isolate (LR-2) were selected. The criteria for selection required antibiotics in combination to belong to lower generation with simple mechanism of action, and low FICI values. To further confirm the synergistic mechanism of action, Time-Kill assays were performed on selected combinations according to a formerly described protocol [27] (Figure 3). Briefly, MHB was inoculated with test organism and incubated to midlog phase. Aliquots containing antibiotic combination (two or three drug combination) and test MRSA at density of $10^{6} \mathrm{CFUs}$ were incubated at $37^{\circ} \mathrm{C}$. At times $0 \mathrm{~h}, 2 \mathrm{~h}, 4 \mathrm{~h}, 6 \mathrm{~h}$, and $8 \mathrm{~h}$, the inoculum was withdrawn, serially diluted from $10^{3}$ to $10^{7}$, and $100 \mu \mathrm{l}$ of each dilution was plated on a nutrient agar medium and incubated at optimal growth condition of $S$. aureus to determine colony counts [27]. Killing kinetics were interpreted as "synergistic" when $\geq 2-\log 10$ reduction in growth was observed in the colony forming units (CFU) at $24 \mathrm{~h}$ with antibiotic combination compared to most active drug alone, and "indifferent" when <2-log10 decrease was observed.

Synergistic drug combinations were then tested on other randomly selected methicillin-resistant $S$. aureus isolates, and their MIC and FICI values were determined to validate drug combination synergy.

All assays were conducted in duplicate, and mean MIC values were calculated along with standard deviations. The FICI values were calculated from the mean MIC values obtained in individual and combination assays. The standard deviation is represented in graphs on the error bars.

\section{Results}

Results obtained from the Kirby-Bauer disk diffusion method on MRSA isolate (LR-2) showed complete resistance to penicillin, cephalosporin, fluoroquinolones, aminoglycosides, and glycopeptides and partial resistance to carbapenem, tetracycline, and certain miscellaneous agents. Therefore, MRSA isolate (LR-2) was classified as XDR [28]. Individual MIC assays showed insensitivity to all antibiotics from Table 1 except for imipenem $(\mathrm{MIC}=4 \mu \mathrm{g} / \mathrm{ml})$. Double and triple antibiotic combinations from diverse subclasses were tested against MRSA LR-2 to identify the combinations that were synergistic when administered concomitantly. Figure 4 shows the interactions between antibiotics indicating synergistic or indifferent relationship. 
TABLE 1: List of antibiotics tested against the MRSA isolate (LR-2).

\begin{tabular}{|c|c|c|c|c|c|}
\hline Antibiotic & Brand name & Manufacturer & Form & Potency & Final conc. \\
\hline \multicolumn{6}{|l|}{ Cephalosprin } \\
\hline Cephradine (RAD) & Velosef & GlaxoSmithKline (GSK) & IV & $1 \mathrm{~g}$ & $10 \mathrm{mg} / \mathrm{ml}$ \\
\hline Cefuroxime (CXM) & Zinacef & GlaxoSmithKline (GSK) & IV & $1.5 \mathrm{~g}$ & $10 \mathrm{mg} / \mathrm{ml}$ \\
\hline Ceftazidime (CAZ) & Fortum & GlaxoSmithKline (GSK) & IV & $500 \mathrm{mg}$ & $10 \mathrm{mg} / \mathrm{ml}$ \\
\hline Cefotaxime (CTX) & Claforan & Sanofi-Aventis Pak Ltd. & IV & $1 \mathrm{~g}$ & $10 \mathrm{mg} / \mathrm{ml}$ \\
\hline Ceftriaxone (CRO) & Rocephin & F.Hoffmann-La Roche Ltd. & Tablets & $1 \mathrm{~g}$ & $10 \mathrm{mg} / \mathrm{ml}$ \\
\hline Cefepime (FEP) & Maxipime & GlaxoSmithKline (GSK) & IV & $1 \mathrm{~g}$ & $10 \mathrm{mg} / \mathrm{ml}$ \\
\hline \multicolumn{6}{|l|}{ Aminoglycosides } \\
\hline Amikacin (AK) & Gracil & Sami Pharmaceticals (Pvt.) Ltd. & IV & $500 \mathrm{mg} / 2 \mathrm{ml}$ & $10 \mathrm{mg} / \mathrm{ml}$ \\
\hline Gentamycin $(\mathrm{CN})$ & Genticyn & Ray Pharma (Pvt.) Ltd. & IV & $80 \mathrm{mg} / 2 \mathrm{ml}$ & $10 \mathrm{mg} / \mathrm{ml}$ \\
\hline Tobramycin (TOB) & Nebcin & AGP Limited & IV & $80 \mathrm{mg} / 2 \mathrm{ml}$ & $10 \mathrm{mg} / \mathrm{ml}$ \\
\hline Fosfomycin (FOS) & Monural & Zambon & Sachet & $3 \mathrm{~g}$ & $10 \mathrm{mg} / \mathrm{ml}$ \\
\hline \multicolumn{6}{|l|}{ Fluoroquinolones } \\
\hline Ofloxacin (OFX) & Oflobid & Hilton Pharma (Pvt.) Ltd. & Tablets & $200 \mathrm{mg}$ & $10 \mathrm{mg} / \mathrm{ml}$ \\
\hline Levofloxacin (LVX) & Leflox & Getz Pharma & Tablets & $500 \mathrm{mg}$ & $10 \mathrm{mg} / \mathrm{ml}$ \\
\hline Sparfloxacin (SPX) & Sparaxin & Abbot Laboratories (Pak) Ltd. & Tablets & $100 \mathrm{mg}$ & $10 \mathrm{mg} / \mathrm{ml}$ \\
\hline Moxifloxacin (MOX) & Avelox & Bayer Pharma AG & & & $10 \mathrm{mg} / \mathrm{ml}$ \\
\hline \multicolumn{6}{|l|}{ Carbapenem } \\
\hline Meropenem (MEM) & Meronem & Pfizer Limited & IV & $500 \mathrm{nmg}$ & $10 \mathrm{mg} / \mathrm{ml}$ \\
\hline Imipenem (IPM) & Tienam & Merck Sharp \& Dohme Corp. (MSD) & IV & $500 \mathrm{mg}$ & $10 \mathrm{mg} / \mathrm{ml}$ \\
\hline \multicolumn{6}{|l|}{ Ampicillin $+\beta$-lactam Inhibitor } \\
\hline Amoxicillin/clavulanic acid (AUG) & Augmentin & GlaxoSmithKline (GSK) & Tablets & $625 \mathrm{mg}$ & $10 \mathrm{mg} / \mathrm{ml}$ \\
\hline Piperacillin/tazobactam (TZP) & Tanzo & Bosch Pharmaceuticals (Pvt.) Ltd & IV & $4.5 \mathrm{~g}$ & $10 \mathrm{mg} / \mathrm{ml}$ \\
\hline
\end{tabular}

All pairwise combinations of cephalosporin with aminoglycosides or carbapenem did not exhibit a synergistic interaction. However, when combined with fluoroquinolones, some synergy was observed especially with moxifloxacin $\quad($ MOX/RAD $\quad F I C I=0.140 ; \quad$ MOX/CXM FICI $=0.265 ; \quad \mathrm{MOX} / \mathrm{CAZ} \quad \mathrm{FICI}=0.140 ; \quad \mathrm{MOX} / \mathrm{CTX}$ FICI $=0.265$ ) (Figure $1(b)$ ). It must be noted that moxifloxacin is a higher generation antibiotic than levofloxacin [29]. An interesting pairwise synergistic combination of cephalosporin and fluoroquinolone with low FICI (0.281) was observed between CAZ/LVX (both lower generation antibiotics) (Figure 1(c)). All double antibiotic combinations of carbapenem were unsuccessful except for when combined with fluoroquinolones. Meropenem showed synergy with higher generation of fluoroquinolones (sparfloxacin and moxifloxacin with FICI of 0.132 and 0.256 , respectively) (Figure 1(d)), and fluoroquinolones-imipenem combination showed a completely synergistic interaction $(\mathrm{FICI}=0.126)$ (Figure 1(e)). All pairwise combinations with aminoglycosides were indifferent.

Triple combination of amoxicillin/clavulanic acid with cephalosporin yielded a peculiar result when synergy was observed with cephradine ( $1^{\text {st }}$ generation cephalosporin) with an FICI value of 0.281 (Figure 2(b)). This finding was interesting because if proven successful in further experimentation, an ineffective lower generation antibiotic [30] can be brought in use again. Sensitivity was observed when amoxicillin/clavulanic acid was combined with fluoroquinolones (FICI 0.25) (Figure 2(c)) and aminoglycosides $($ FICI $=0.125)$ (Figure 2(d)). Triple combinations of piperacillin/tazobactam with moxifloxacin showed synergy $(\mathrm{FICI}=0.281)$ (Figure 2(e)), and with aminoglycosides, namely, tobramycin $(\mathrm{FICI}=0.281)$ and fosfomycin $($ FICI $=0.281) \quad($ Figure 2(f)), piperacillin/tazobactam, and meropenem combination also showed synergy $(\mathrm{FICI}=0.375)$.

The combinations selected for further testing included levofloxacin-ceftazidime; amoxicillin/clavulanic acidtobramycin; amoxicillin/clavulanic acid-cephradine; amoxicillin/clavulanic acid-ofloxacin; and piperacillin/tazobactam-tobramycin. Levofloxacin combination with cephalosporin was selected instead of moxifloxacin combination because it is a lower-generation gluoroquinolone. Amoxicillin/clavulanic acid and cephradine was selected because both antibiotics other than the inhibitors are of lower generation. Amoxicillin/clavulanic acid showed FICI values lower than 0.5 with fluoroquinolones and aminoglycosides, so one combination from each group was selected. Ofloxacin in combination to amoxicillin/clavulanic acid made this list because it is the lowest-generation aminoglycoside. However, tobramycin though is not a low generation aminoglycoside but showed good potential with other penicillin- $\beta$-lactam inhibitor combinations. Hence, tobramycin in amalgamation with amoxicillin/clavulanic acid and with piperacillin-tazobactam was selected.

Time-Kill kinetics illustrate up to 8 -fold reduction in growth compared to most active drug alone which means a synergistic relation exists between levofloxacin-ceftazidime; amoxicillin/clavulanic acid-tobramycin; and piperacillintazobactam-tobramycin (Figures 3(a)-3(e)). Bactericidal effect was observed within 8 hours of incubation in all three combinations.

The combination showing $>2-\log 10$ reduction when tested on other randomly selected MRSA isolates, 


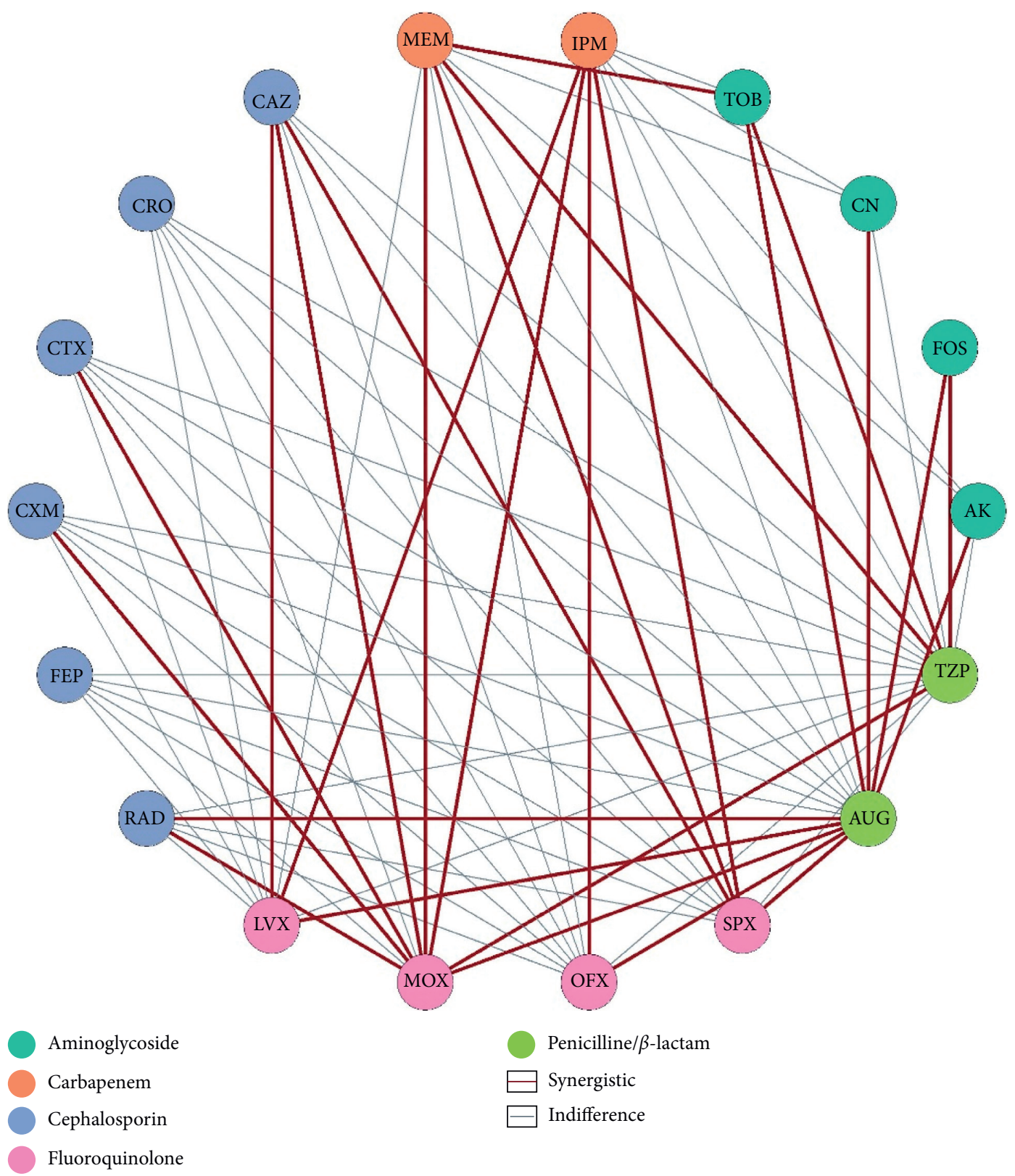

FIGURE 1: Pairwise antibiotic testing against MRSA. (a) List of pairwise combinations. (b) MIC by broth microdilution indicates that synergistic relationship exists between moxifloxacin and lower-generation cephalosporin (FICI 0.14-0.265). (c) Levofloxacin-ceftazidime shows FICI value as low as 0.281 indicating synergy. (d) Meropenem demonstrates synergy with higher generation of fluoroquinolones, i.e., moxifloxacin (FICI 0.132) and sparfloxacin (FICI 0.265). (e) Imipenem shows collateral sensitivity with all fluoroquinolones (FICI 0.126 each).

levofloxacin-ceftazidime combination, was the most successful combination because collateral sensitivity was observed in $80 \%$ of MRSA isolates. Both amoxicillin/clavulanic acid-tobramycin; piperacillin-tazobactam-tobramycin combination showed synergy in 50\% MRSA isolates.

\section{Discussion}

Rapid emergence of extensive drug resistance in Grampositive and Gram-negative bacteria necessitates identification of novel therapeutic approaches. Reliance on monoantibiotic therapeutic approach provided selection pressure for development of resistance. With the depleting arsenal of antibiotics and a gap in the successful development of alternative therapeutics, determining synergistic combination of currently available antibiotics could provide an effective alternative to curtailing the alarming increase in antibiotic resistance [31].

The purpose of this study was to find combinations from diverse subclasses of antibiotics that can produce synergistic effects in combinations against extremely drug-resistant MRSA isolates. We report that a novel combination of levofloxacin-ceftazidime (LVX/CAZ) acts synergistically to produce bactericidal effect on XDR MRSA isolate LR-2. 


\begin{tabular}{|c|}
\hline $\begin{array}{l}\text { Amoxicillin/clavulanic } \\
\text { acid }\end{array}$ \\
\hline Cephalosporin \\
\hline $\begin{array}{l}\text { Cephradine } \\
\text { Cefuroxime } \\
\text { Ceftazidime } \\
\text { Cefotaxime } \\
\text { Ceftriaxone } \\
\text { Cefepime }\end{array}$ \\
\hline Aminoglycoside \\
\hline $\begin{array}{l}\text { Amikacin } \\
\text { Gentamycin } \\
\text { Tobramycin } \\
\text { Fosfomycin }\end{array}$ \\
\hline Fluoroquinolone \\
\hline $\begin{array}{l}\text { Ofloxacin } \\
\text { Levofloxacin } \\
\text { Sparfloxacin } \\
\text { Moxifloxacin }\end{array}$ \\
\hline Carbapenem \\
\hline $\begin{array}{l}\text { Imipenem } \\
\text { Meropenem }\end{array}$ \\
\hline Piperacillin/tazobactam \\
\hline Cephalosporin \\
\hline $\begin{array}{l}\text { Cephradine } \\
\text { Cefuroxime } \\
\text { Ceftazidime } \\
\text { Cefotaxime } \\
\text { Ceftriaxone } \\
\text { Cefepime }\end{array}$ \\
\hline Aminoglycoside \\
\hline $\begin{array}{l}\text { Amikacin } \\
\text { Gentamycin } \\
\text { Tobramycin } \\
\text { Fosfomycin }\end{array}$ \\
\hline Fluoroquinolone \\
\hline $\begin{array}{l}\text { Ofloxacin } \\
\text { Levofloxacin } \\
\text { Sparfloxacin } \\
\text { Moxifloxacin }\end{array}$ \\
\hline Carbapenem \\
\hline $\begin{array}{l}\text { Imipenem } \\
\text { Meropenem }\end{array}$ \\
\hline
\end{tabular}

(a)

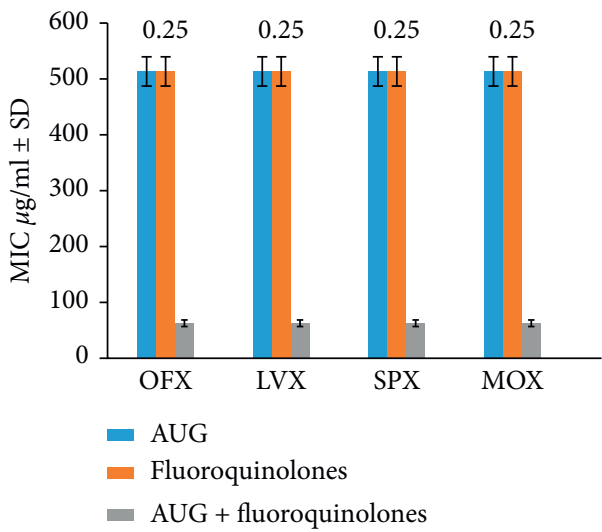

(c)

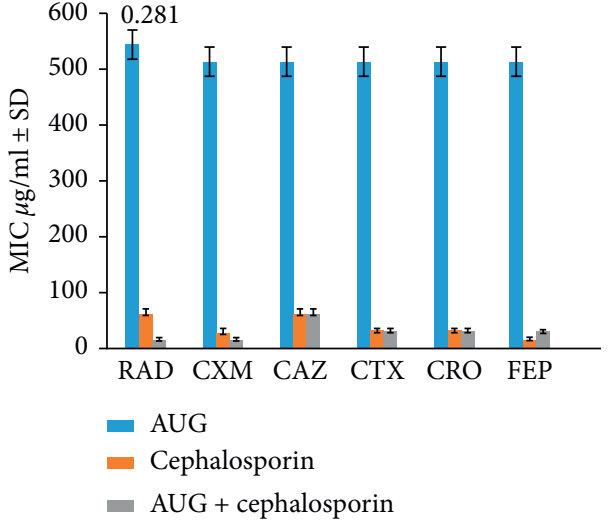

(b)

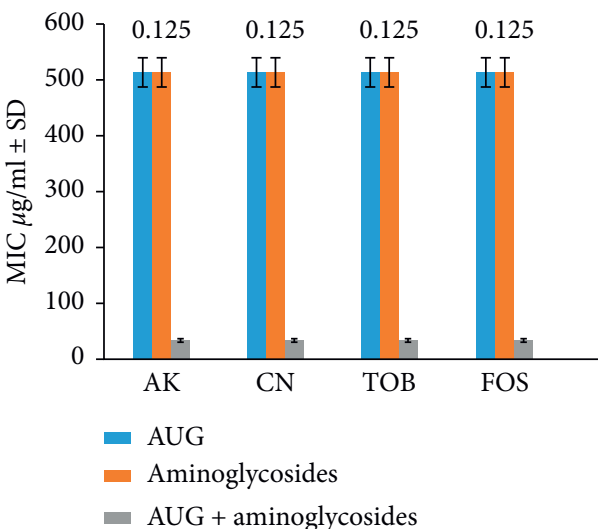

(d)

Figure 2: Continued. 


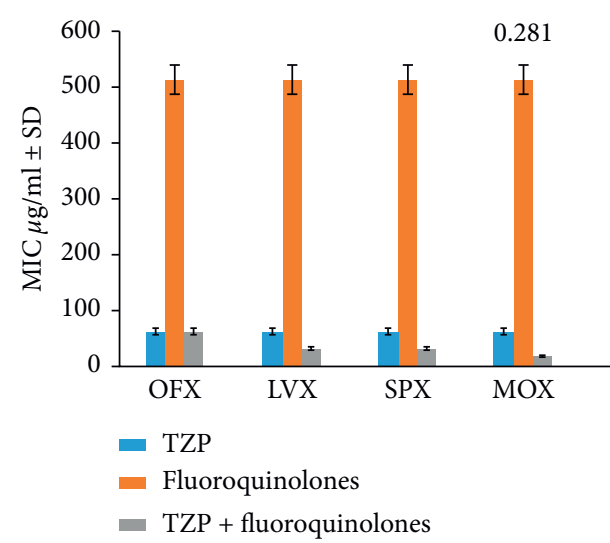

(e)

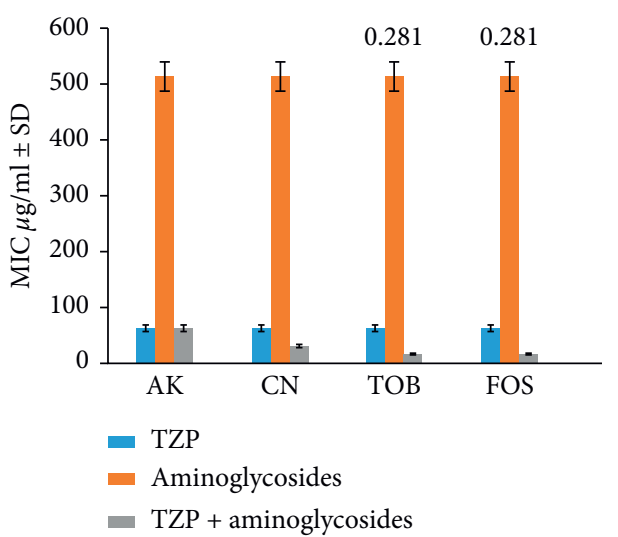

(f)

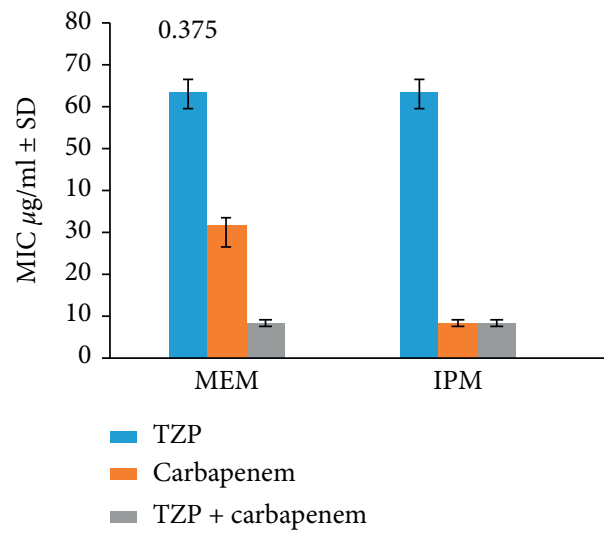

(g)

Figure 2: Triple antibiotic testing against MRSA. (a) List of triple combinations. (b) Amoxicillin-clavulanic acid-cephalosporin MIC indicates that synergistic relationship exists only between amoxicillin-clavulanic acid-cephradine (FICI 0.281). (c) MIC of amoxicillinclavulanic acid-fluoroquinolone combination shows that synergy exists in all combinations (FICI 0.25 each). (d) A significant decrease in MIC of amoxicillin-clavulanic acid-aminoglycoside in combinations (FICI 0.125). (e) Reduction in combined MIC is only observed with moxifloxacin in piperacillin-tazobactam-fluoroquinolone combinations (FICI 0.281). (f) Significant activity is observed when piperacillintazobactam is combined with higher-generation aminoglycoside, i.e., tobramycin (FICI 0.281) and fosfomycin (FICI 0.281). (g) Meropenem in combination with piperacillin-tazobactam exhibits synergy (FICI 0.375).

Previously, fluoroquinolone-cephalosporin combination was shown to have synergistic effect against $P$. aeruginosa [32].

Our findings on pairwise combinations suggest that fluoroquinolones (especially levofloxacin and moxifloxacin) are very effective when combined with cephalosporin and carbapenem antibiotics. Though monotherapy with fluoroquinolones is recommended to be minimized for treatment of $S$. aureus infections because of multidrug resistance [33], this finding provides a solution to overcome fluoroquinolone resistance in MRSA.

The overall higher sensitivity ratios to amoxicillin/ clavulanic acid in combination with other antibiotics is not surprising since existing evidence on antimicrobial combinations suggests that despite being the first-line antibiotic, beta-lactam antibiotics in combination therapy are crucial for the treatment of obstinate $S$. aureus bacteremia [34]. Besides, amoxicillin/clavulanic acid is recommended in amalgamations to overcome vancomycin or daptomycin resistance in MRSA [35-37]. Amoxicillin/clavulanic acid in conjunction with cephradine, an ineffective lower-generation antibiotic against MRSA [30], was an interesting combination. This lower-generation antibiotic proved successful in Time-Kill studies and shows the potential to be used again as a therapeutic agent in combination. Although, amoxicillin/clavulanic acid-tobramycin trio showed $>2$ $\log 10$ decrease in XDR MRSA isolate (LR-2), it did not show synergistic interactions in other isolates. Amoxicillin/clavulanic acid/fluoroquinolone (ciprofloxacin) combination has also been proven successful before for treatment of Gram-negative infections of prosthetic joints [38]. However, it must be noted that most of these isolates showed low individual MIC values for tobramycin and amoxicillin/ clavulanic acid, and so the FICI values were mostly "indifferent." Synergy between piperacillin-tazobactam-meropenem combination is consistent with the findings of PR Gonzales and his colleagues [20].

The inability to confirm all synergistic combinations by Time-Kill assay was a limitation of our study. Due to this, we might have overlooked certain combinations that might 


\begin{tabular}{|c|c|}
\hline \multicolumn{2}{|c|}{ Fluoroquinolone + cephalosporin } \\
\hline Ofloxacin & Cephradine \\
\hline Levofloxacin & Cefuroxime \\
\hline Sparfloxacin & Ceftazidime \\
\hline \multirow[t]{3}{*}{ Moxifloxacin } & Cefotaxime \\
\hline & Ceftriaxone \\
\hline & Cefepime \\
\hline \multicolumn{2}{|c|}{ Fluoroquinolone + aminoglycoside } \\
\hline Ofloxacin & Amikacin \\
\hline Levofloxacin & Gentamycin \\
\hline Sparfloxacin & Tobramycin \\
\hline Moxifloxacin & Fosfomycin \\
\hline \multicolumn{2}{|c|}{ Cephalosporin + aminoglycoside } \\
\hline Cephradine & Amikacin \\
\hline Cefuroxime & Gentamycin \\
\hline Ceftazidime & Tobramycin \\
\hline Cefotaxime & Fosfomycin \\
\hline Ceftriaxone & \\
\hline Cefepime & \\
\hline \multicolumn{2}{|c|}{ Aminoglycoside + carbapenem } \\
\hline Amikacin & Imipenem \\
\hline Gentamycin & Meropenem \\
\hline Tobramycin & \\
\hline Fosfomycin & \\
\hline \multicolumn{2}{|c|}{ Carbapenem + cephalosporin } \\
\hline Imipenem & Cephradine \\
\hline \multirow[t]{5}{*}{ Meropenem } & Cefuroxime \\
\hline & Ceftazidime \\
\hline & Cefotaxime \\
\hline & Ceftriaxone \\
\hline & Cefepime \\
\hline \multicolumn{2}{|c|}{ Fluoroquinolone + carbapenem } \\
\hline Ofloxacin & Imipenem \\
\hline Levofloxacin & Meropenem \\
\hline Sparfloxacin & \\
\hline Moxifloxacin & \\
\hline
\end{tabular}

(a)

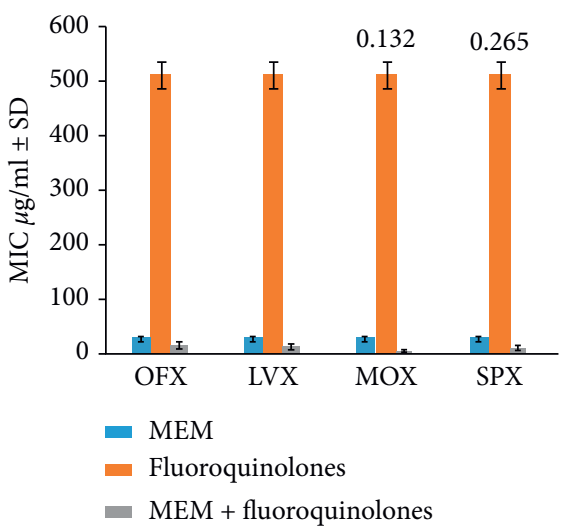

(d)
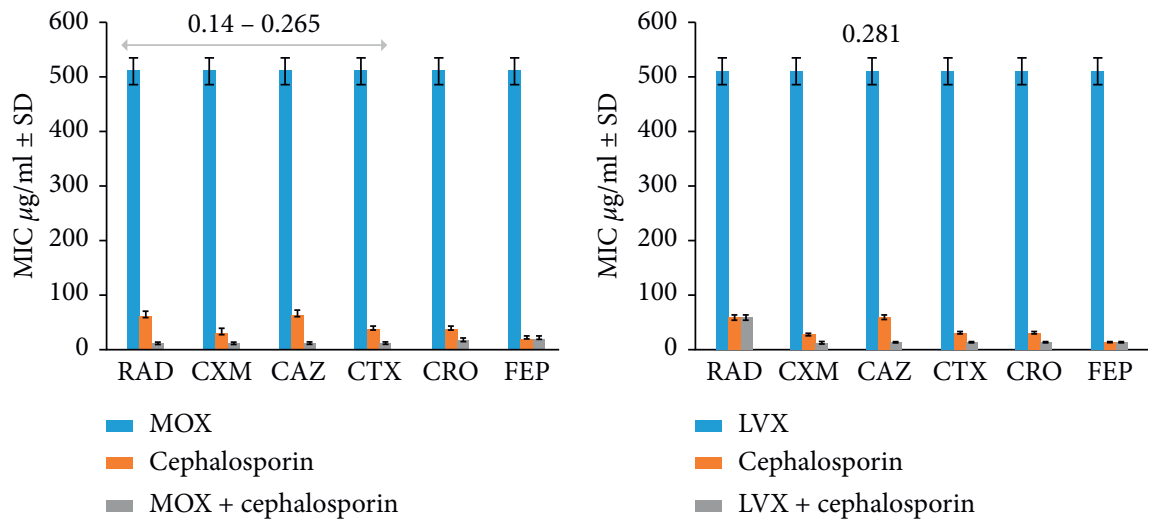

(b)

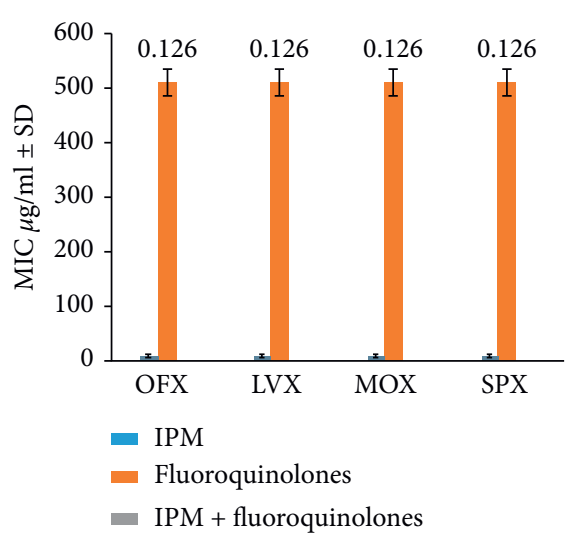

(e)

Figure 3: Time-Kill kinetics on antibiotics individually and collaterally. (a) Levofloxacin-ceftazidime combination with 8-fold growth reduction compared to most active monotherapy. (b) <2-log 10 difference in killing with amoxicillin-clavulanic acid-cephradine combination therapy compared to most active single treatment shows lack of synergy. (c) Amoxicillin-clavulanic acid-ofloxacin combination is collaterally indifferent as decrease in bacterial growth is $<2-\log 10$. (d) Amoxicillin-clavulanic acid-tobramycin combination shows synergy because of $>2-\log 10$ growth reduction. (e) Collateral sensitivity is shown in piperacillin-tazobactam-tobramycin combination.

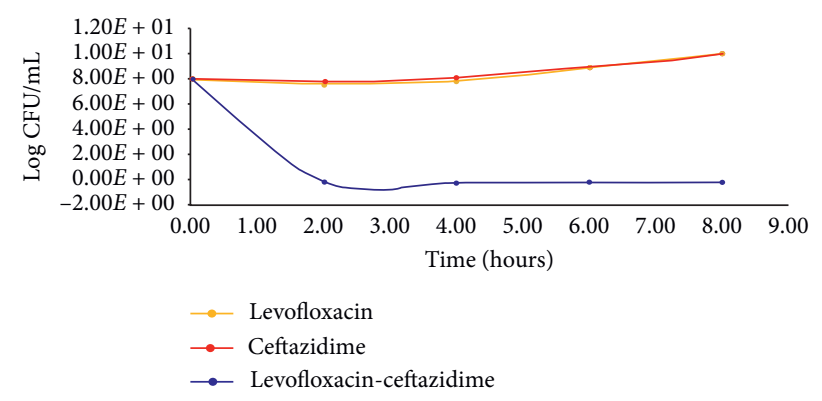

(a)

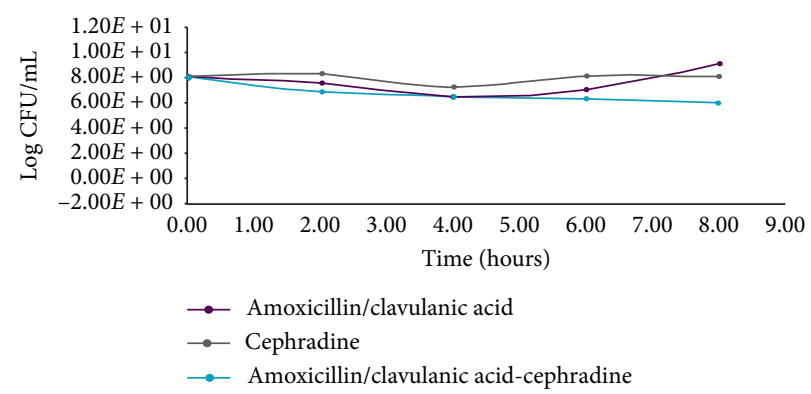

(b)

Figure 4: Continued. 


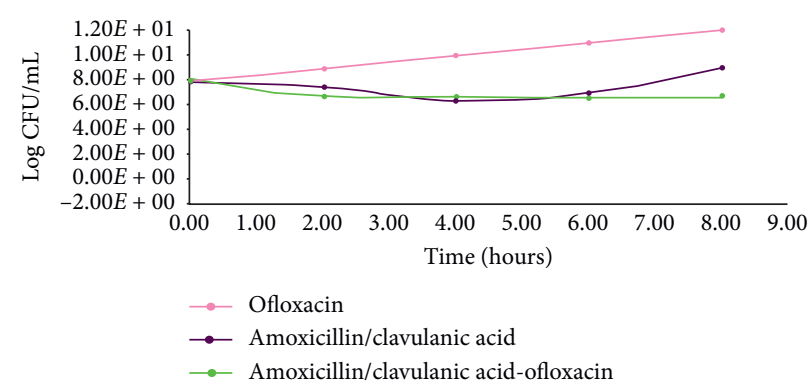

(c)

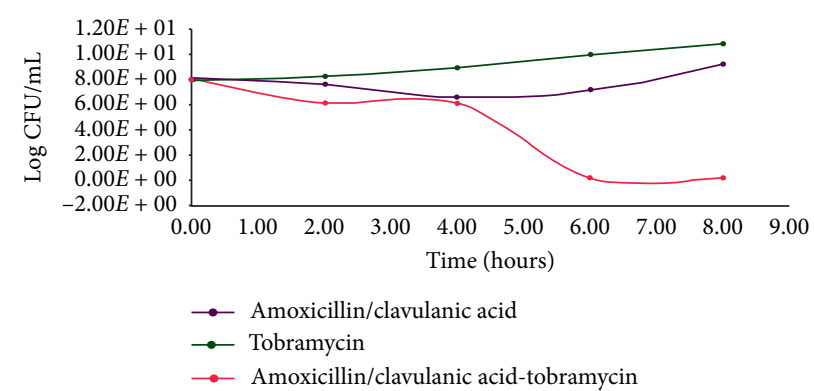

(d)

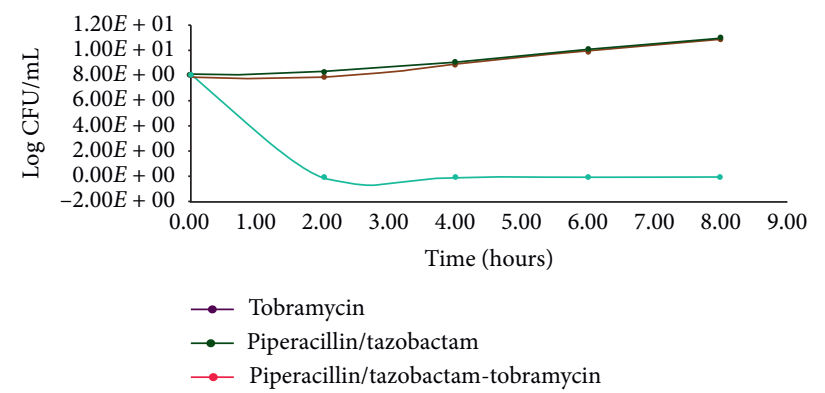

(e)

FIGURE 4: Collateral relationship network of antibiotic combinations from diverse subclasses on MRSA LR-2.

produce better results than the ones we screened. However, the main objective of our study to test and identify combinations from diverse subclasses that interacted synergistically, belonged to lower generations, produced bactericidal effects, and overcome the resistance for first-line antibiotics that could not be used anymore was achieved.

Although coadministration of drugs is a promising approach to mitigate rapid evolution of antibiotic resistance, it may also lead to production of unexpected and unwanted outcomes as reported previously [39]. Although the FIC index was determined to ensure that a synergistic relationship exists between selected antibiotics when used in combination, further investigations may help optimize the management of antibiotic combinations.

\section{Conclusion}

We report that pairwise LVX/CAZ combination allows us to use first-line antibiotics that not only produce bactericidal effects against XDR MRSA but also address need of time by extending the lifespan of existing antibiotics. This combination will be immediately available for use since these drugs have already been approved by the FDA. Resistance to antibiotic combination though inevitable, evidence-based synergistic studies might diminish the emergence of resistance. For future prospects, we propose that these combinations should be further tested in vivo to determine if these amalgams are as effective in vivo as they were in vitro. We also suggest that investigations should be carried out on how these combinations, where target proteins of the antibiotics involved in amalgam, work synergistically at molecular and systemic level to give a better insight. This will help predict better and robust combinations in future.

\author{
Abbreviations \\ MRSA: Methicillin-resistant Staphylococcus aureus \\ XDR: Extremely drug resistant \\ VRE: Vancomycin-resistant Enterococcus species \\ FDA: Food and Drug Administration \\ MIC: Minimum inhibitory concentration \\ FICI: Fractional inhibitory concentration index \\ MHB: Muller-Hinton Broth \\ CLSI: Clinical and Laboratory Standards Institute \\ RAD: Cephradine \\ CXM: Cefuroxime \\ CAZ: Ceftazidime \\ CTX: Cefotaxime \\ CRO: Ceftriaxone \\ FEP: Cefepime \\ AK: Amikacin \\ $\mathrm{CN}$ : Gentamycin \\ TOB: Tobramycin \\ FOS: Fosfomycin \\ OFX: Ofloxacin \\ LVX: Levofloxacin \\ SPX: Sparfloxacin \\ MOX: Moxifloxacin \\ MEM: Meropenem \\ IPM: Imipenem \\ AUG: Amoxicillin/clavulanic acid \\ TZP: Piperacillin/tazobactam.
}

\section{Data Availability}

Data produced and analyzed during this study are included in this article. 


\section{Conflicts of Interest}

The authors declare no conflicts of interest.

\section{Authors' Contributions}

SN conducted the research and prepared the manuscript. MA collected the samples. SV and US carried out the study. DG revised and reviewed the study. SA and KM designed, reviewed, and approved the research.

\section{Acknowledgments}

This work was partially funded by the United States Agency for International Development award (3220-29047) to Saadia Andleeb, Gautham Dantas, and Carey-Ann D. Burnham. The authors would like to thank the tertiary care hospitals for providing them bacterial isolates. This work was supported by Pak-US S\&T Coop. Program Phase VI.

\section{References}

[1] R. P. Wenzel, M. D. Nettleman, R. N. Jones, and M. A. Pfaller, "Methicillin-resistant Staphylococcus aureus: implications for the 1990s and effective control measures," The American Journal of Medicine, vol. 91, no. 3, pp. S221-S227, 1991.

[2] D. Cipolla, M. Giuffrè, C. Mammina, and G. Corsello, "Prevention of nosocomial infections and surveillance of emerging resistances in NICU," The Journal of Maternal-Fetal \& Neonatal Medicine, vol. 24, no. sup1, pp. 23-26, 2011.

[3] N. A. Turner, B. K. Sharma-Kuinkel, S. A. Maskarinec et al., "Methicillin-resistant Staphylococcus aureus: an overview of basic and clinical research," Nature Reviews Microbiology, vol. 17, no. 4, pp. 203-218, 2019.

[4] H. Wisplinghoff, T. Bischoff, S. M. Tallent, H. Seifert, R. P. Wenzel, and M. B. Edmond, "Nosocomial bloodstream infections in US hospitals: analysis of 24,179 cases from a prospective nationwide surveillance study," Clinical Infectious Diseases, vol. 39, no. 3, pp. 309-317, 2004.

[5] B. J. Wiggli, R Frei, R Laffer, S Tschudin Sutter, and A. F Widmer, "Survival from methicillin-sensitive Staphylococcus aureus bloodstream infections over 20 years: a cohort of 1328 patients," Swiss Medical Weekly, vol. 147, no. 3940, Article ID w14508, 2017.

[6] D. C. Oliveira, C. Milheiriço, and H. de Lencastre, "Redefining a structural variant of staphylococcal cassette chromosome mec, SCCmec type VI," Antimicrobial Agents and Chemotherapy, vol. 50, no. 10, pp. 3457-3459, 2006.

[7] A. S. Lee et al., "Methicillin-resistant Staphylococcus aureus," Nature Reviews Disease Primers, vol. 4, no. 1, pp. 1-23, 2018.

[8] H. Chambers, "The changing epidemiology of Staphylococcus aureus?" Emerging Infectious Diseases, vol. 7, no. 2, pp. 178-182, 2001.

[9] J. Liu, D. Chen, B. M. Peters et al., "Staphylococcal chromosomal cassettes mec (SCCmec): a mobile genetic element in methicillin-resistant Staphylococcus aureus," Microbial Pathogenesis, vol. 101, pp. 56-67, 2016.

[10] W. A. McGuinness, N. Malachowa, and F. R. DeLeo, "Focus: infectious diseases: vancomycin resistance in Staphylococcus aureus," The Yale Journal of Biology and Medicine, vol. 90, no. 2, pp. 269-281, 2017.
[11] L. K. Yaw, J. O. Robinson, and K. M. Ho, "A comparison of long-term outcomes after meticillin-resistant and meticillinsensitive Staphylococcus aureus bacteraemia: an observational cohort study," The Lancet Infectious Diseases, vol. 14, no. 10, pp. 967-975, 2014.

[12] Y. Bhusal, C. M. Shiohira, and N. Yamane, "Determination of in vitro synergy when three antimicrobial agents are combined against Mycobacterium tuberculosis," International Journal of Antimicrobial Agents, vol. 26, no. 4, pp. 292-297, 2005.

[13] Y. Cai, D. Chai, R. Wang, B. Liang, and N. Bai, "Colistin resistance of Acinetobacter baumannii: clinical reports, mechanisms and antimicrobial strategies," Journal of Antimicrobial Chemotherapy, vol. 67, no. 7, pp. 1607-1615, 2012.

[14] K. Goderska, S. Agudo Pena, and T. Alarcon, "Helicobacter pylori treatment: antibiotics or probiotics," Applied Microbiology and Biotechnology, vol. 102, no. 1, pp. 1-7, 2018.

[15] T. Tängdén, M. Karvanen, L. E. Friberg, I. Odenholt, and O. Cars, "Assessment of early combination effects of colistin and meropenem againstPseudomonas aeruginosaandAcinetobacter baumanniiin dynamic time-kill experiments," Infectious Diseases, vol. 49, no. 7, pp. 521-527, 2017.

[16] J. R. Lenhard, R. L. Nation, and B. T. Tsuji, "Synergistic combinations of polymyxins," International Journal of Antimicrobial Agents, vol. 48, no. 6, pp. 607-613, 2016.

[17] L. M. Baddour, W. R. Wilson, A. S. Bayer et al., "Infective endocarditis in adults: diagnosis, antimicrobial therapy, and management of complications," Circulation, vol. 132, no. 15, pp. 1435-1486, 2015.

[18] S. E. Cosgrove, G. A. Vigliani, M. Campion et al., "Initial lowdose gentamicin forStaphylococcus aureusBacteremia and endocarditis is nephrotoxic," Clinical Infectious Diseases, vol. 48, no. 6, pp. 713-721, 2009.

[19] M. N. Bulloch, L. Paumen, S. Eure, and J. G. Stewart, "Impact of higher vancomycin troughs on vancomycin-induced nephrotoxicity," Infectious Diseases in Clinical Practice, vol. 25, no. 4, pp. 203-208, 2017.

[20] P. R. Gonzales, M. W. Pesesky, R. Bouley et al., "Synergistic, collaterally sensitive $\beta$-lactam combinations suppress resistance in MRSA," Nature Chemical Biology, vol. 11, no. 11, pp. 855-861, 2015.

[21] J. Hudzicki, "Kirby-Bauer disk diffusion susceptibility test protocol," 2009.

[22] I. Wiegand, K. Hilpert, and R. E. W. Hancock, "Agar and broth dilution methods to determine the minimal inhibitory concentration (MIC) of antimicrobial substances," Nature Protocols, vol. 3, no. 2, pp. 163-175, 2008.

[23] Y. Cai, R. Wang, F. Pei, and B.-B. Liang, "Antibacterial activity of allicin alone and in combination with $\beta$-lactams against Staphylococcus spp. and Pseudomonas aeruginosa," The Journal of Antibiotics, vol. 60, no. 5, pp. 335-338, 2007.

[24] V. Amorese, M. Donadu, D. Usai et al., "In vitro activity of essential oils against Pseudomonas aeruginosa isolated from infected hip implants," The Journal of Infection in Developing Countries, vol. 12, no. 11, pp. 996-1001, 2018.

[25] A. Bua, D. Usai, M. G. Donadu et al., "Antimicrobial activity of Austroeupatorium inulaefolium (H.B.K.) against intracellular and extracellular organisms," Natural Product Research, vol. 32, no. 23, pp. 2869-2871, 2018.

[26] A. Pinna et al., "In vitro antimicrobial activity of a new ophthalmic solution containing hexamidine diisethionate 0.05\% (keratosept)," Cornea, vol. 39, no. 11, pp. 1415-1418, 2020.

[27] M. M. Sopirala, J. E. Mangino, W. A. Gebreyes et al., "Synergy testing by Etest, microdilution checkerboard, and time-kill 
methods for pan-drug-resistant Acinetobacter baumannii," Antimicrobial Agents and Chemotherapy, vol. 54, no. 11, pp. 4678-4683, 2010.

[28] A.-P. Magiorakos, A. Srinivasan, R. B. Carey et al., "Multidrug-resistant, extensively drug-resistant and pandrug-resistant bacteria: an international expert proposal for interim standard definitions for acquired resistance," Clinical Microbiology and Infection, vol. 18, no. 3, pp. 268-281, 2012.

[29] D. C. Hooper, "Mechanisms of action and resistance of older and newer fluoroquinolones," Clinical Infectious Diseases, vol. 31, no. Supplement_2, pp. S24-S28, 2000.

[30] K. Farzana et al., "Bactericidal activity of various brands of cephradine against Staphylococcus aureus," International journal of physical sciences, vol. 6, pp. 1501-1507, 2011.

[31] D. Usai, M. Donadu, A. Bua et al., "Enhancement of antimicrobial activity of pump inhibitors associating drugs," The Journal of Infection in Developing Countries, vol. 13, no. 2, pp. 162-164, 2019.

[32] D. N. Fish, M. K. Choi, and R. Jung, "Synergic activity of cephalosporins plus fluoroquinolones against Pseudomonas aeruginosa with resistance to one or both drugs," Journal of Antimicrobial Chemotherapy, vol. 50, no. 6, pp. 1045-1049, 2002.

[33] N. D. Gade and M. S. Qazi, "Fluoroquinolone therapy in Staphylococcus aureus infections: where do we stand?" Journal of Laboratory Physicians, vol. 5, no. 2, pp. 109-112, 2013.

[34] R. Bartash and P. Nori, "Beta-lactam combination therapy for the treatment of Staphylococcus aureus and Enterococcus species bacteremia: a summary and appraisal of the evidence," International Journal of Infectious Diseases, vol. 63, pp. 7-12, 2017.

[35] D. Kaur and S. Chate, "Study of antibiotic resistance pattern in methicillin resistant Staphylococcus aureus with special reference to newer antibiotic," Journal of Global Infectious Diseases, vol. 7, no. 2, p. 78, 2015.

[36] J. M. Entenza, M. Giddey, J. Vouillamoz, and P. Moreillon, "In vitro prevention of the emergence of daptomycin resistance in Staphylococcus aureus and enterococci following combination with amoxicillin/clavulanic acid or ampicillin," International Journal of Antimicrobial Agents, vol. 35, no. 5, pp. 451-456, 2010.

[37] S. Jamil, U. Saad, and S. Hafiz, "Can amoxicillin clavulanate be used for treating MRSA," Journal of Pharmacology and Medicinal Chemistry, vol. 1, no. 1, pp. 21-23, 2017.

[38] C. A. Aboltins, M. M. Dowsey, K. L. Buising et al., "Gramnegative prosthetic joint infection treated with debridement, prosthesis retention and antibiotic regimens including a fluoroquinolone," Clinical Microbiology and Infection, vol. 17, no. 6, pp. 862-867, 2011.

[39] A. Aszalos, "Drug-drug interactions affected by the transporter protein, P-glycoprotein (ABCB1, MDR1): II. Clinical aspects," Drug Discovery Today, vol. 12, no. 19-20, pp. 838-843, 2007. 